\title{
Eye Avoidance of Threatening Facial Expressions in Parents of Children with ASD
}

\author{
Tingting Yang ${ }^{1, *}$ \\ Dandan $\mathrm{Li}^{1-3, *}$ \\ Yifan Zhang' \\ Long Zhang ${ }^{2,3}$ \\ Hong $\mathrm{Li}^{4}$ \\ Gong-Jun $\mathrm{ji}^{1-3}$ \\ Zhenhai Yang ${ }^{5}$ \\ Lei Zhang ${ }^{1,3}$ \\ Chunyan Zhu',3,* \\ Kai Wang ${ }^{1-3,6}$
}

'School of Mental Health and Psychological Sciences, Anhui Medical University, Hefei, 230022, People's Republic of China;

${ }^{2}$ Department of Neurology, the First Affiliated Hospital of Anhui Medical

University, Hefei, 230022, People's Republic of China; ${ }^{3}$ Anhui Province Key Laboratory

of Cognition and Neuropsychiatric

Disorders, Hefei, 230022, People's Republic

of China; ${ }^{4}$ Department of Neurological

Rehabilitation of Children, Anhui Provincial Children's Hospital, Hefei, 230022, People's Republic of China; ${ }^{5}$ Department of Rehabilitation therapy, The First Clinical Medical College of Anhui Medical University, Hefei, 230022, People's Republic of China; 'Institute of Artificial Intelligence, Hefei Comprehensive National Science

Center, Hefei, 230022, People's Republic of China

*These authors contributed equally to this work

Correspondence: Lei Zhang; Chunyan

Zhu

School of Mental Health and Psychological Sciences, Anhui Medical University, Hefei,

People's Republic of China

Email zhangleil7236@aliyun.com;

ayswallow@I26.com
Objective: Previous research found that autism spectrum disorder (ASD) was characterized by eye avoidance of threatening facial expressions. However, it still remains unclear as to whether these abnormalities are present in parents of children with ASD. Our study aimed to investigate the gaze patterns of parents of children with ASD in the threatening facial expressions.

Methods: Thirty-four parents of children with ASD and 35 parents of typically developing (TD) children participated in our study. We investigated the total fixation time of participants when they viewed different facial expression (eg, happy, fearful, angry, sad) videos and examined changes in the fixation duration over time.

Results: We observed the following: a) the total fixation time of the parents of children with ASD on the eyes of fearful faces was significantly shorter than that of the normal group, and the difference lasted for five seconds (four to six seconds, eight to nine seconds) throughout the process; and b) The parents of children with ASD avoided the eyes of angry expression faces at around five seconds after the stimulus onset.

Conclusion: We concluded that parents of children with ASD tended to avoid the eyes of threatening expression faces while viewing the dynamic emotions video.

Keywords: parents of children with ASD, total fixation time, threatening facial expressions

\section{Introduction}

Autism spectrum disorder (ASD) is a complex neurodevelopmental disorder, the main characteristics of which include developmental deficits in social interactions and communication as well as restricted interests and behaviors. ${ }^{1-3}$ Twin studies and largescale epidemiological studies provide clear evidence of the genetic components of ASD, and it is estimated that the disease heritability of ASD is as high as $90 \%$, which means that ASD is one of the most heritable neuropsychiatric disorders. ${ }^{2,4,5}$ However, another study indicated that the heritability in ASD was $52.4 \%$, which was largely attributable to common mutations. ${ }^{6}$ This shows that genetic and environmental factors play an important role in the pathophysiological mechanism of ASD. Family members of those with ASD share some of the behavioral and cognitive features of ASD. ${ }^{5,7,8}$ A considerable number of parents and siblings of children with ASD exhibit behavioral characteristics that are similar to certain core symptoms of ASD. ${ }^{9}$ These definite characteristics of ASD are more prevalent among family members of those with ASD, and they are termed the "broad autism phenotype". 10,11

Eye avoidance was considered as the most striking feature of ASD. ${ }^{12-14}$ The gaze-aversion hypothesis holds that those with ASD will not avoid looking at the 
eyes of all emotional faces, but that they will avoid looking at threatening faces. This aversion to looking at threatening faces can be understood as an attempt to alleviate the discomfort that is experienced while gazing at the eyes of threatening faces, and the eyes are regarded as the most powerful tool to convey threatening information. ${ }^{15,16}$ Fear and anger are perceived as threatening emotions, and both of these emotions tend to elicit a strong and rapid neurological response. However, they differ in their nature. For instance, eye avoidance in ASD was the most salient phenomenon when looking at threatening facial expressions (eg, anger). ${ }^{17}$ Anger expresses information that communicates a kind of direct threat, and it indicates that the threat originates from the figure that corresponds to the face. As such, it conveys the presence of a type of social threat. Adults with ASD exhibit poorer recognition abilities and a reduction in attention to others' eyes when observing fearful facial expressions. ${ }^{18}$ Fearful facial expressions communicate an indirect threat, and they indicate that the threat derives from the surrounding environment, which means that they can convey a type of environmental threat. ${ }^{19}$

For first-degree relatives of ASD, data from functional magnetic resonance imaging studies (fMRI) revealed that the ASD group, as well as siblings of those in this group, had less activation of the fusiform gyrus (a brain area involved in facial processing) compared to those in the normally-developed control group. ${ }^{20}$ Moreover, parents of children with ASD showed a remarkable reduction in the time spent fixating the eye region of faces. ${ }^{21,22}$ Nevertheless, thus far, few studies have investigated facial expressions that are perceived as threatening among relatives of individuals with ASD. Only one study of siblings of children with ASD suggested that these infants showed a bias towards fearful facial expressions, and a stronger fear bias could relate to less social difficulty. ${ }^{23}$ It remains unclear as to whether parents of children with ASD exhibit the same abnormalities as those observed in the case of ASD when processing threatening facial expressions, and whether this abnormality is associated with social disorders.

Eye-tracking (ET) has also been widely used in studies of gaze patterns and attention processes in individuals with ASD while viewing socially salient stimuli, such as emotional facial expressions. ${ }^{24}$ In the current study, the experimental material included dynamic emotional face processing videos. In social interactions, humans must constantly monitor the facial expressions of others so that they can then adjust and adapt their behavior accordingly. People's facial expressions are dynamic in nature. Because of the complexity of the analytical method, many experimental designs of emotional facial expressions in ASD focused on presenting static basic emotions, and few studies employed dynamic stimuli by incorporating authentic naturalistic elements. ${ }^{25-27}$ Nevertheless, by comparing the performance of people with ASD on static and dynamic emotional stimuli, previous studies concluded that static stimuli could not accurately reflect the needs of daily social interactions, and dynamic emotional stimuli proved more authentic as they accurately reflected the process of emotional changes, and were deemed more suitable for distinguishing between two groups of emotional differences. ${ }^{28,29}$ At present, dynamic videos are rarely used to study gaze patterns when attending to emotional faces among parents of children with ASD.

The current study aimed to test whether eye avoidance differences occurred in parents of children with ASD in respect to four facial expressions (ie, happy, angry, sad, and fearful). We hypothesized that eye avoidance in parents of children with ASD specifically targets threat-related facial expressions, and the greater the aversion to looking at the eyes of threatening faces, the more serious the social disorder. However, previous eye movement studies have mostly focused on the overall viewing time of first-degree relatives of $\mathrm{ASD} .{ }^{18,30}$ Clinical observations and daily life indicate that people with ASD tend to react by taking the initiative and avoiding faces after they have perceived the faces, ${ }^{31-33}$ and the gaze pattern of ASD first-degree relatives is similar to that of ASD. Therefore, the overall viewing time may not be able to reveal the characteristics of emotional face processing in ASD parents. This requires a more detailed time analysis of the entire mechanism underlying face processing, which could elucidate differences observed between parents of ASD children and the normal group in respect to gaze patterns on the eye region of emotional faces. We expected that the parents of children with ASD would not avoid looking at the eyes of threatening faces during the early stage of the video, but that they would avoid looking at the eyes of threatening faces during the middle and later stages of the video.

\section{Methods}

\section{Participants}

We recruited 38 parents (12 males, 26 females) whose children had been diagnosed with ASD by pediatric 
psychiatrists, according to the diagnostic criteria of ASD published in the Diagnosis and Statistics Manual of Mental Disorders (DSM-V). Four adults were subsequently excluded, due to technical issues during data recording ( $\mathrm{n}$ $=1$ ), or eye-tracking calibration failure $(n=3)$. Ultimately, our study included 34 participants (10 males, 24 females), aged between 21 and 49 years of age $(\mathrm{M}=34.61, \mathrm{SD}=$ 6.61). A total of 35 participants, who were matched for sex, age, were recruited as controls (12 males, 23 females; age range $28-40 ; \mathrm{M}=33.89 ; \mathrm{SD}=3.67$ ). In addition, the ASD parent groups and control groups were matched on the basis of their intelligence level (ASD parents, $27.17 \pm 1.45$; controls, $26.41 \pm 2.08$; Montreal Cognitive Assessment). The demographic characteristics of the participants are reported in Table 1. None of the participants had any previous history of psychiatric problems, or severe physical or eye problems, and all of the participants were right-handed. All participants volunteered to participate in this experiment and provided their written informed consent.

\section{Apparatus}

We used the SMI-RED portable ET recording system (iView X Hi-Speed, Senso Motoric Instruments, Berlin, Germany) to monitor participants' eye movements. ET includes a host display and a subject-specific display. The screen resolution was set to $1680 \times 960$ pixels, the sampling frequency was $250 \mathrm{~Hz}$, and the spatial resolution was $0.03^{\circ}$. We used Experiment Center software for the ET experiments and BeGaze software for data analysis.

\section{Materials}

\section{Montreal Cognitive Assessment}

The current study utilized the final revised version of the Montreal Cognitive Assessment (MoCA) scale. This is a rating tool for rapid screening for cognitive impairment. It includes 11 items that cover eight cognitive fields, such as attention and centralized executive function, memory, language, visual structure skills, abstract thinking, computation and orientation. The scale is used to measure the level of intelligence, with a total score of 30 points.

\section{Autism-Spectrum Quotient (AQ)}

The AQ test is used as a quantitative measurement tool for broader autism phenotypes (BAP). It was first proposed and compiled by Baron-Cohen and his colleagues in 2001, and later translated and adapted by Zhang Long. In addition, the reliability and validity of the adapted AQ scale has been verified. ${ }^{34}$ The AQ test is a self-report screening tool that measures the severity of autism characteristics using five sub-scales (ie, social skills, communication, attention to detail, attention shift, and imagination). Higher scores are indicative of the ASD prototype.

\section{Interpersonal Ability Scale (IAS)}

The Interpersonal Ability Scale was compiled by a Chinese scholar, Ma Jianqing, and its reliability and validity have been verified. It is used to measure social interaction skills. The scale uses a five-point scoring method and has a total score of 150 points. Higher scores indicate reduced social skills.

\section{Eye-Tracking Paradigm}

The ET paradigm used in this study was adapted from a previous study. ${ }^{35}$ We used a total of four films, which conveyed four basic emotions, ie, happiness, sadness, anger, and fear. Each film consisted of the same scene, with a woman standing in front of a black background. Only the woman's head and shoulders were shown in these films. The scenes of each film were identical (see Figure 1). All of the films were silent. No story and no speech were presented on the computer screen. The computer screen only showed a woman who dynamically and frankly expressed her feelings (10 seconds each). In the happy film, the woman laughed heartily. In the fear

Table I Demographic Data of ASD Parent and TD Parent (Mean \pm SD)

\begin{tabular}{|l|c|c|c|c|}
\hline & ASD Parents & TD Parents & t/ $\chi$ & p-value \\
\hline Subject(n) & 34 & 35 & $/$ & 1 \\
\hline Sex (male/female) & $10 / 24$ & $12 / 23$ & 0.67 & 0.42 \\
\hline Age & $34.61 \pm 6.61$ & $33.89 \pm 3.67$ & 0.57 & 0.57 \\
\hline Years of education & $12.61 \pm 2.84$ & $13.40 \pm 3.22$ & -0.88 & 0.41 \\
\hline MOCA & $27.17 \pm 1.45$ & $26.41 \pm 2.08$ & 1.64 & 0.20 \\
\hline
\end{tabular}

Abbreviations: ASD parent, parent of children with ASD; TD parent, parent of typical development children. 


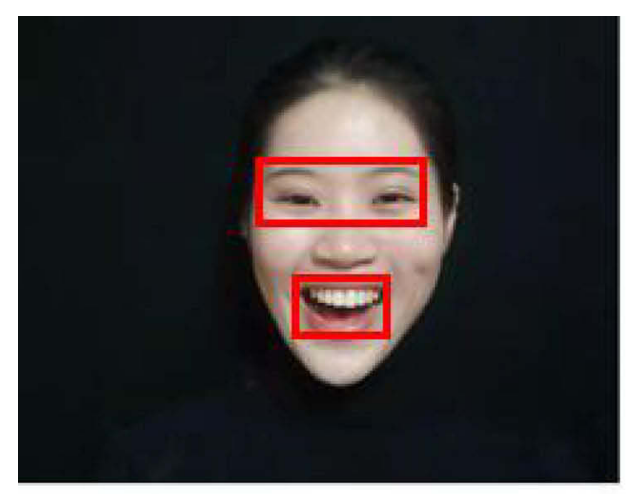

happy

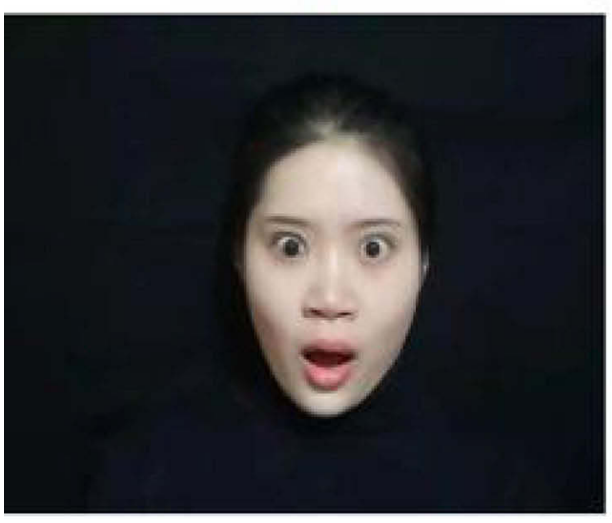

fear

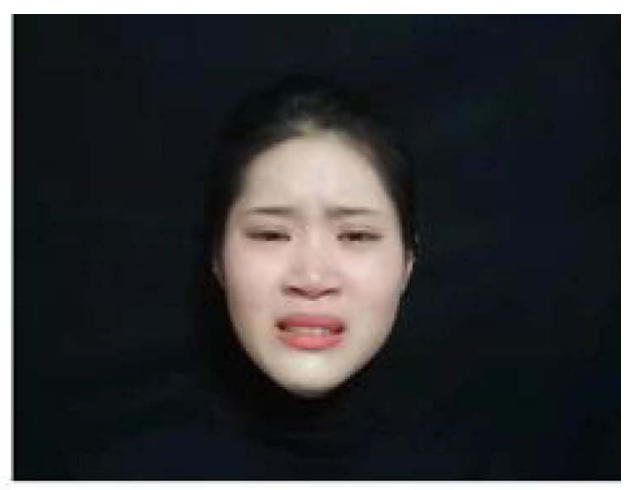

sad

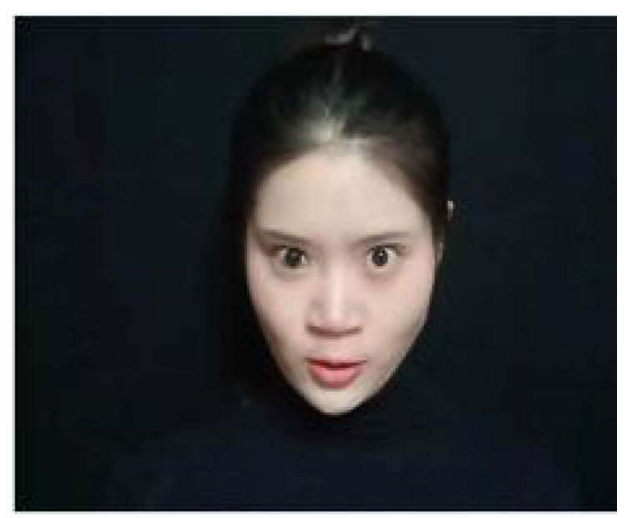

anger

Figure I Four emotional videos and AOls.

film, she expressed fear by communicating extremely scared facial expressions. In the angry film, the woman expressed her anger directly by biting her teeth and widening her eyes. In the sad film, the woman cried tears.

\section{Design and Procedure}

All of the participants were assessed individually in a silent room with an adjustable chair and a 30 -inch computer on a table Participants were seated $60-80 \mathrm{~cm}$ directly in front of the screen with their eyes looking up at the center of the screen. Before the experiment, we used the SMI-RED five-point calibration method for each participant. We included participants in the experimental study only if all five points (ie, average error of each calibration point was less than $1 \circ$ of visual angle) for the two eyes were captured by the SMI-RED. A trial began with a picture showing the instruction "Please watch the following film carefully." Then, the eyetracker randomly played four films, and participants were requested to watch randomly. After the participants had finished watching the films, the message "Thank you for your cooperation" appeared on the screen.

\section{Data Analyses}

The current study used the participants' total fixation time while watching emotional films as an indicator. For each film used in the experiment, regions of interest were delimited manually using SMI-RED eye-tracking software. We defined two areas of interest (AOIs) on the actress's face in the film, ie, the eyes and mouth (see Figure 1). It has been suggested that the eyes and mouth play a crucial role in emotion recognition, and a combination of both of these regions has been recommended. ${ }^{36}$ Finally, the eye-tracker counted all of the data in each of the AOIs. We then used BeGaze analysis software to export eye movement data. The BeGaze software generated a variable termed "Total Fixation Time," which is the sum of the duration of all fixations within the given AOIs. 
This study used SPSS version 16.0 software to analyze the data. Demographic data and scale data were analyzed using two independent sample $t$-tests and chi-square tests. A 2 (Group: ASD parent and TD parent) $\times 4$ (Expression: happy, sad, fearful and angry) repeated-measures analysis of variance (ANOVA) was used to measure whether the two groups showed significant differences in the AOI fixation time for the four different expressions. If a significant interaction was found between "group" and "expression", we decided that we would carry out Post hoc comparisons (after Bonferroni correction) to determine whether eye avoidance in parents of children with ASD was modulated by different expressions.

We furthermore employed temporal-course analyses to explore how the eye-avoidance pattern of parents of children with ASD changed with time, and how the eye-gazing time of each group changed over time. To track the time course of the eye movements, each 10 -second video presentation was subdivided into 10 one-second intervals. A 2 (Group: ASD parent and TD parent) $\times 10$ (Time:1-10s) repeated-measures ANOVA was conducted for four expressions (ie, happy, sad, fearful, and angry) in this study.

\section{Results}

\section{Behavioral results}

For the IAS, the results of the independent samples $t$-test showed that the scores of ASD parents were significantly higher than those of parents in the control group $(F=2.11$, $p=0.05)$. For the AQ test, the results of the independent samples $t$-test showed that no significant differences were observed between the two groups in respect to the AQ scale total score and social skills $(F=0.00, p=0.99)$, attention to conversion $(F=0.72, p=0.40)$, attention to detail $(F=0.29 . p=0.60)$, and imagination $(F=0.07, p=$ 0.79) sub-scale scores, whereas ASD parents scored significantly higher on the speech sub-scale scores $(F=1.31$, $p=0.26$ ) than normal parents. (see Table 2).

\section{Eye-Tracking Results Total Fixation Time on Eyes}

In this study, a 2 (Group: ASD parents and TD parents) $\times$ 4 (Expression: happy, sad, fearful, and angry) repeatedmeasures ANOVA was performed to examine group differences in total fixation time on the eyes. As shown in Figure $2 \mathrm{~A}$, the analysis revealed a main effect of expression on the total fixation time $(F=17.52, p<0.01)$, while no significant main effect of group was observed $(F=$ 2.59, $p=0.11)$. In addition, we found a Group $\times$ Expression interaction $(F=2.85, p=0.04)$. Post hoc comparisons (after Bonferroni correction) were performed to detect whether the differences in the total fixation time between the two groups were modulated by different facial expressions. The results showed that the fixation time of the parents of children with ASD on the eyes of fearful faces was shorter than that of the control group $(F=5.75$, $p=0.02)$. Furthermore, no difference was found in the fixation time between the two groups for the video clips that depicted happiness $(F=0.88, p=0.35)$, anger $(F=$ $0.73, p=0.39)$ and sadness $(F=0.74, p=0.39)$.

We also performed post hoc pairwise $t$-tests (after Bonferroni correction). The results revealed that the parents of children with ASD paid less attention to eyes of sad faces than to the eyes of fearful faces $(t=3.10, p=0.01)$. No difference was observed between the fixation time on eyes of angry and fearful faces $(t=0.56, p>0.99)$, and no difference

Table 2 Comparison of IAS and AQ Date Between ASD Parent and TD Parent (Mean \pm SD)

\begin{tabular}{|l|l|l|l|l|}
\hline & ASD Parents & TD Parents & $t$ & P-value \\
\hline IAS & $93.47 \pm 18.58$ & $85.31 \pm 13.19$ & -1.99 & $0.05^{*}$ \\
\hline AQ & $114.12 \pm 9.98$ & $111.46 \pm 9.27$ & 1.11 & -0.02 \\
\hline Social skills & $21.70 \pm 4.19$ & $21.71 \pm 3.53$ & 0.85 & 0.26 \\
\hline Attention shift & $25.55 \pm 2.78$ & $24.91 \pm 3.31$ & -0.53 & 0.99 \\
\hline Attention to detail & $22.79 \pm 3.67$ & $23.29 \pm 3.99$ & 2.34 & 0.40 \\
\hline Communication & $22.85 \pm 4.14$ & $20.49 \pm 4.17$ & 0.27 & 0.60 \\
\hline Imagination & $21.24 \pm 2.71$ & $21.06 \pm 2.88$ & & 0.77 \\
\hline
\end{tabular}

Note: ${ }^{*} \mathrm{P}<0.05$.

Abbreviations: ASD parent, parent of children with ASD; TD parent, parent of typical development children; IAS, Interpersonal Ability Scale; AQ, Autism-Spectrum Quotient. 

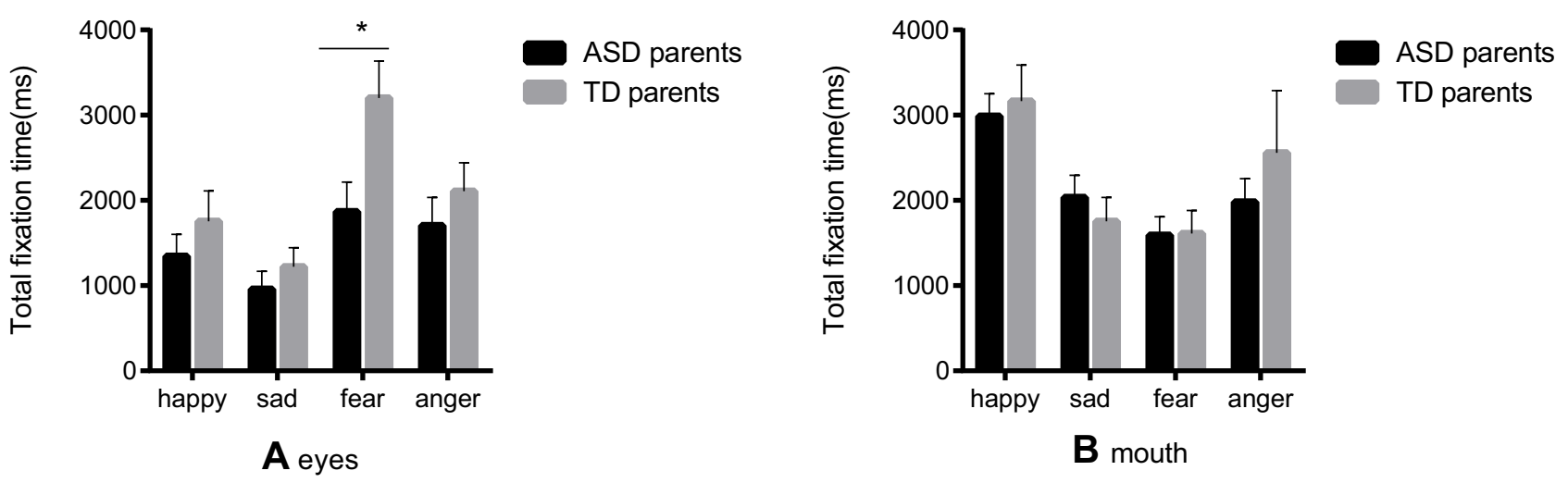

Figure 2 Total fixation time on the eyes $(\mathbf{A})$ and mouth $(\mathbf{B})$ of different emotional faces of the ASD parents and the TD parents (error bars denote standard errors; *Denotes $p<0.05$ )

was found in the case of happy and sad faces $(t=0.732, p=$ $0.470)$, happy and angry faces $(t=1.22, p>0.99)$, happy and fearful faces $(t=1.78, p=0.46)$, and sad and angry faces $(t=$ $2.54, p=0.07)$. The control group was associated with a significantly longer fixation time on the eyes of fearful faces than on the eyes of sad $(t=6.85, p<0.01)$, happy ( $t=$ $5.00, p<0.01)$, angry faces $(t=3.79, p<0.01)$, and the fixation time on the eyes of angry faces was also longer than that observed in the case of sad faces $(t=3.06, p=0.02)$. We found no difference between happy and sad faces $(t=1.85$, $p=0.40)$, and happy and angry faces $(t=1.21, p>0.99)$.

\section{Temporal-Course Analysis}

In addition to analyzing the fixation time on the eyes during the entire viewing time, we further examined how the eyefixation duration of each facial expression changed over time and determined when each group avoided eye contact. The results are shown in Figure 3. For the fearful face, the main effect of time was significant $(t=30.66, p<0.01)$, the main effect of group was significant $(t=2.02, p=0.04)$, and the interaction effect was not significant $(t=1.03, p=0.41)$. In order to analyze the influence of time on the fixation time of the two groups, we conducted Post hoc comparisons, the results of which are as follows: The parents of children in the ASD group spent less time looking at eyes during the two periods of four to six seconds and eight to nine seconds compared with the control group $(p<0.05)$. For the angry face, the main effect of time was significant $(t=6.41$, $p=0.01)$, the main effect of group was significant $(t=2.23$, $p=0.02$ ), and the interaction effect was not significant ( $t=0.57, p=0.82)$. Post hoc comparisons indicated that the eye fixation time of the two groups was quite similar for all periods except at the fifth second $(p<0.05)$. For the sad and happy faces, there were no significant differences in time, group, and interaction factors. In addition, we found that the fixation time on eyes was similar in all epochs between two groups. On the other hand, during the early stages (1st to $3 \mathrm{rd}$ seconds) of the presentation of the four emotional faces, no significant difference was found in the fixation time between the two groups. (see Figure 3).

\section{Total Fixation Time on Mouth}

For fixation time on the mouth, the results of the repeatedmeasures ANOVA showed that the main effect of emotion was statistically significant $(F=18.37, p<0.01)$, although no significant main effect of group was found $(F=0.21$, $p=0.648)$, and the interaction between groups and emotion types was also not statistically significant $(F=0.64$, $p=0.59)$. For the four film clips, the results of the ANOVA revealed no significant difference between the two groups in respect to the fixation time on the actor's mouth. (see Figure 2B).

\section{Discussion}

In the present study, we observed that eye-avoidance in parents of children with ASD was most evident in the case of fearful facial expressions. Moreover, the temporalcourse analysis showed that, for fearful faces, the parents of children with ASD spent less time looking at the eyes than parents in the control group, and this difference occurred significantly in the two periods of 4 to 6 seconds and 8 to 9 seconds; for angry facial expressions, it was only at the fifth second of the video that the parents of children with ASD avoided gazing at their eyes, and eye avoidance was not observed at other times. For the happy and sad facial expressions, group differences were rarely observed at any time. In addition, the behavioral results showed that the parents of children with ASD had 

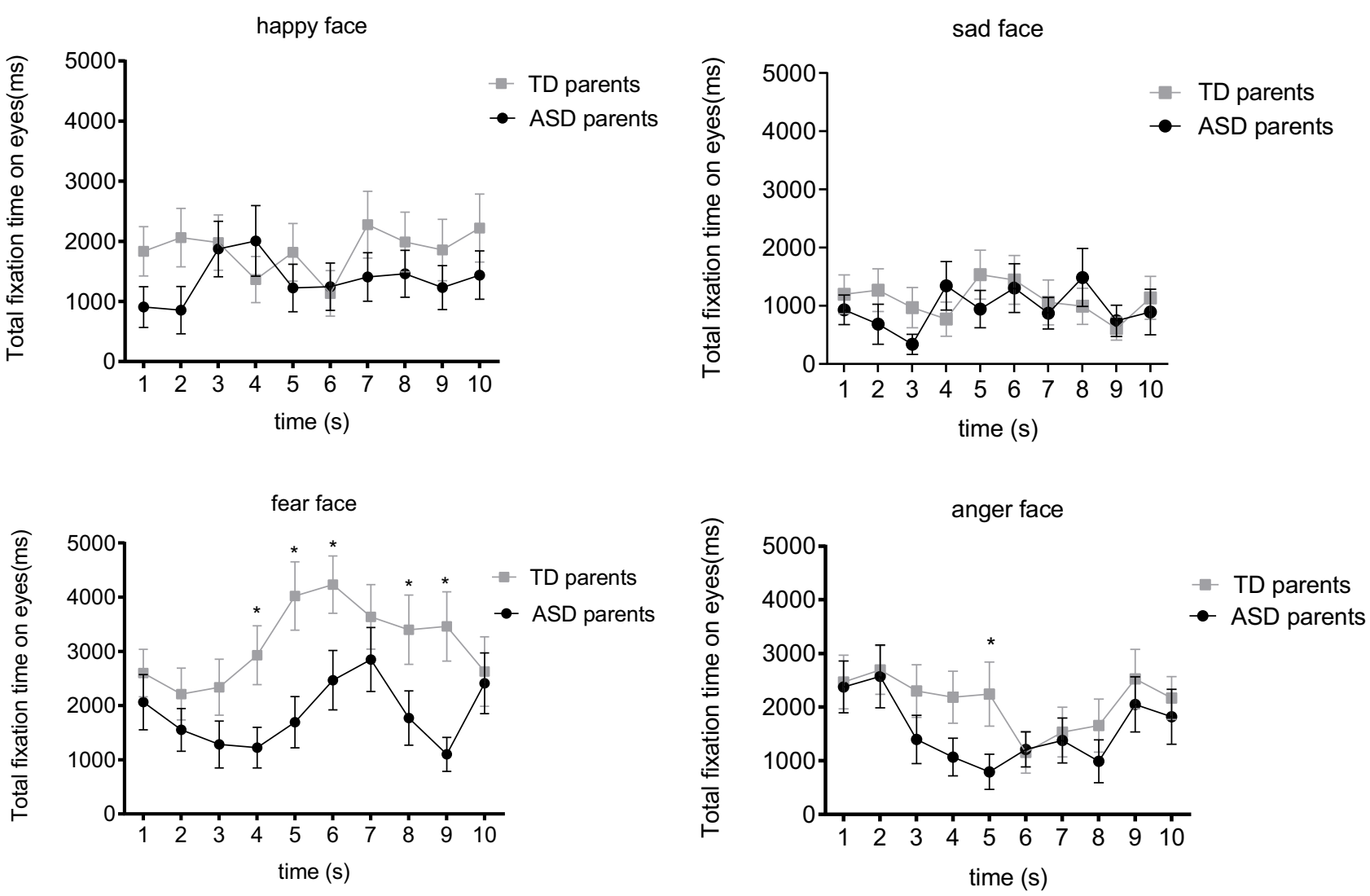

Figure 3 Eye-fixation time of the parents of children with ASD and the control groups for different expressions over time (error bars denote standard errors; *Denotes $p<0.05)$.

significantly higher IAS scores than parents of children in the control group. For the AQ test, although the total score was not significantly different, significant differences were observed between the two groups with regard to the communication sub-scales.

Fear and anger are emotions that are perceived as threats. As such, they tend to elicit a strong, rapid nervous response. ${ }^{37}$ Our research proved that eye-avoidance among parents of children with ASD occurred when they viewed fearful faces at the middle and late stages of the video clips, which showed that perceived environmental threats were more likely to cause gaze aversion in parents of children with ASD. For the angry faces, although the overall difference in time between the groups was not significant, the temporal-course analysis indicated that eye-avoidance occurred at 5 seconds (in the middle stages of the video clip) after the onset of the angry face stimulus. Parents of children with ASD showed less fixation specificity to the eyes of the angry expression face, which may mirror their tendency to avoid the socially threatening discomfort that is aroused by angry facial expressions. In addition, we found no significant differences in respect to the early stages of viewing the emotional video clips. This may be because the initial stage of viewing emotional faces is the stage at which emotions are recognized, and many studies of people with autistic characteristics and first-degree relatives with ASD demonstrated abnormalities in the emotion recognition abilities of these groups. ${ }^{38-40}$ Our research is consistent with previous studies, which may indicate that parents with ASD may be able to recognize dynamic threatening emotions that are a closer representation of the real world. Once they identify threatening information, they will choose to avoid looking at the threatening information.

A crucial result of our research is that the gaze pattern of parents of children with ASD was different to the gaze pattern of normal parents, and this difference was evidenced by a reduction in the fixation time on the eyes of threatening faces. Increased attention to threat-related information in the environment is critical for survival. Fearful and angry facial expressions communicate threatening stimuli and attract attention faster than emotionally positive facial expressions. ${ }^{41,42}$ Numerous studies have proved that the eyes are the core organs of the face that 
convey emotions, and they are the central features of a threatening face, as they can communicate social and threatening messages. ${ }^{43-45}$ Our conclusions suggested that eye-avoidance among parents of children with ASD occurred when viewing the eyes of a threatening face, and the reasons for this observation may include the following: (1) Parents of children with ASD may be less alert, and this manifests in the form of insufficient attention and an inability to respond quickly to threat-related information in the environment. (2) Directly gazing at threatening eyes may elicit a high level of anxiety, leading to mental and physical discomfort, which may in turn stimulate avoidance behavior.

The amygdala is the core brain area that processes threatening faces. ${ }^{46-48}$ The fMRI studies have revealed dysfunction of the amygdala in persons with ASD and their first-degree relatives. ${ }^{26,44,49,50}$ Our research found that the parents of children with ASD showed abnormalities in their ability to process threatening faces, which may be related to damage to the amygdala. Another finding of our study was that, when viewing threatening faces, the normal control group had a significantly longer gaze time on the eyes of fearful faces than angry faces. Our research results are consistent with previous similar studies, which indicated that, compared with neutral expressions, the perception of fearful and angry expressions enhanced the amygdala's blood-oxygenation-leveldependent response, while fearful expressions seemed to provoke the greatest response. ${ }^{51-53}$ However, few imaging studies directly compared the brain's response to fear and anger, and this response has not been examined in persons with ASD and their first-degree relatives. Therefore, in the case of parents of children with ASD, eye-gaze patterns while viewing threatening faces were inconsistent with the gaze patterns of TD individuals who viewed angry and fearful faces, which may indicate abnormalities in amygdala function. However, this conclusion needs to be confirmed by further research.

In dynamic scenes, the mouth can also convey some social and emotional information. Previous research found that no significant difference between individuals with ASD and TD individuals in respect to the fixation time on the mouth of emotional faces, as the mouth cannot convey more visual and communication information. ${ }^{17}$ Our findings are consistent with the previous study, as no difference was observed in the fixation time between parents of children with ASD and parents of TD children. This may imply that the threatening information transmitted by the mouth is weak. As such, parents of children with ASD do not avoid looking at the mouth area.

In order to examine autistic-like traits and social interaction ability in parents of children with ASD, we conducted IAS and AQ scale tests. For the IAS, we find the lower social interaction ability in parents of children with ASD. However, we did not find a correlation between the eye-avoidance pattern on threatening faces and social interaction skills among the parents of ASD children. Moreover, the results of the AQ test showed that there was no difference in the total score and sub-scales among the parents of children with ASD, except for the communication sub-scale. The correlation analysis suggested that eye-avoidance in parents of children with ASD was not related to autistic-like traits. These results may imply that, when viewing threatening faces, parents' eye-avoidance patterns are not associated with social features of ASD. However, previous studies indicated that the bias towards threatening faces, exhibited by first-degree relatives of people with ASD, was positively correlated with their social skills. ${ }^{23}$ Our research findings are inconsistent with the results of previous studies. This may be explained by the low heterogeneity of the two groups, which may have contributed to the weak correlation between the scale data and eye movement data.

\section{Limitation}

Some of the limitations of this study should be considered when interpreting the results reported here. First, the overall sample size was relatively small, so it is necessary to conduct studies with larger sample sizes to verify our conclusions. Second, our experimental materials included the use of only female facial expressions, and it is unclear whether male faces are perceived as more threatening than female faces. ${ }^{54}$ Finally, neutral emotional faces have not been used as experimental materials in our research. We are not sure whether parents of children with ASD avoid looking at the eyes of neutral faces.

\section{Conclusion}

No study has investigated the gaze characteristics of emotional faces in parents of children with ASD. However, the present study demonstrated how eyetracking measures were able to identify abnormal gaze patterns in parents of children with ASD who gazed less towards the eyes of threatening faces. Therefore, this 
study provided a deeper understanding of the gaze patterns of parents of children with ASD. The most striking feature of our study was that: (1) our experimental materials consisted of dynamic emotional video clips that were more representative of the real world, which may more objectively and accurately reflect the gaze characteristics of participants. (2) Few previous literature publications have studied the two threatening expressions of fear and anger at the same time. Our study examined both of these expressions and concluded that, compared with normal people, the parents of children with ASD tended to avoid looking at the eyes when attending to fearful faces. Our findings have important implications for the future genetics of autism, and they may apply to a number of other neurodevelopmental and psychiatric disorders.

\section{Abbreviations}

ASD, Autism spectrum disorder; ET, Eye-tracking; MoCA, Montreal Cognitive Assessment; IRI, Interpersonal Reactivity Index; AOIs, Areas of interest.

\section{Data Sharing Statement}

The data are not publicly available due to privacy restrictions but are available from the corresponding author on reasonable request.

\section{Ethics Approval and Consent to Participate}

This study was approved by the Research Ethics Committee of the Anhui Medical University. All subjects and their legal representative gave written informed consent. The study adhered to the tenets of the Declaration of Helsinki.

\section{Funding}

This study was supported by the National Natural Science Foundation of China, Grant/Award Number: 82090034, 31970979, 81771456, 31771222, 31800909, 81971689. We would also like to thank International Science Editing (http://www.internationalscienceediting.com) for editing this manuscript.

\section{Disclosure}

The authors report no conflicts of interest in this work.

\section{References}

1. Teresa DB, Noemi M, Arianna B, Paola V. An investigation of attention to faces and eyes: looking time is task-dependent in autism spectrum disorder. Front Psychol. 2018;9:2629. doi:10.3389/ fpsyg.2018.02629

2. Maria C, Kleiman RJ, Chiara MM. Translating genetic and preclinical findings into autism therapies. Dialogues Clin Neurosci. 2017;19 (4):335-343. doi:10.31887/DCNS.2017.19.4/cmanzini

3. Ecker C. The neuroanatomy of autism spectrum disorder: an overview of structural neuroimaging findings and their translatability to the clinical setting. Autism. 2017;21(1):18-28. doi:10.1177/ 1362361315627136

4. Bailey A, Couteur AL, Gottesman I, et al. Autism as a strongly genetic disorder: evidence from a British twin study. Psychol Med. 1995;25(1):63-77. doi:10.1017/S0033291700028099

5. Wilson LB, Tregellas JR, Slason E, Pasko BE, Hepburn S, Rojas DC. Phonological processing in first-degree relatives of individuals with autism: an fMRI study. Hum Brain Mapp. 2013;34(6):1447-1463. doi: $10.1002 / \mathrm{hbm} .22001$

6. Trent G, Lambertus K, Ss J, et al. Most genetic risk for autism resides with common variation. Nat Genet. 2014;46(8):881-885. doi:10.1038/ng.3039

7. Dalton KM, Nacewicz BM, Alexander AL, Davidson RJ. Gazefixation, brain activation, and amygdala volume in unaffected siblings of individuals with autism. Biol Psychiatry. 2007;61(4):512-520. doi:10.1016/j.biopsych.2006.05.019

8. Shivers CM. Empathy and perceptions of their brother or sister among adolescent siblings of individuals with and without autism spectrum disorder. Res Dev Disabil. 2019;92. doi:10.1016/j. ridd.2019.103451

9. Bailey A, Palferman S, Heavey L, Couteur AL. Autism: the phenotype in relatives. J Autism Dev Disord. 1998;28(5):369-392. doi:10.1023/a:1026048320785

10. Piven J, Palmer P, Jacobi D, Childress D, Arndt S. Broader autism phenotype: evidence from a family history study ofmultiple-incidence autism families. Am J Psychiatry. 1997;154 (2):185-190. doi:10.1176/ajp.154.2.185

11. Couteur A, Bailey A, Goode S, et al. A broader phenotype of autism: the clinical spectrum in twins. J Child Psychol Psychiatry. 1996;37 (7):785-801. doi:10.1111/j.1469-7610.1996.tb01475.x

12. Jones W, Klin A. Attention to eyes is present but in decline in 2 6-month-old infants later diagnosed with autism. Nature. 2013;504 (7480):427-431. doi:10.1038/nature12715

13. Rajiv MA, Marcus R, Isabel D, Philipp S. Unconscious avoidance of eye contact in autism spectrum disorder. Sci Rep. 2017;7(1):13378. doi:10.1038/s41598-017-13945-5

14. Król ME, Król M. A novel machine learning analysis of eye-tracking data reveals suboptimal visual information extraction from facial stimuli in individuals with autism. Neuropsychologia. 2019;129. doi:10.1016/j.neuropsychologia.2019.04.022

15. Hutt C, Ounsted C. The biological significance of gaze aversion with particular reference to the syndrome of infantile autism. Behav Sci. 1966;11(5):346-356. doi:10.1002/bs.3830110504

16. Dorit K, Isabel D, Alexander H, Rosa S, Hh R. Atypical reflexive gaze patterns on emotional faces in autism spectrum disorders. $J \quad$ Neurosci. 2010;30(37):12281-12287. doi:10.1523/ JNEUROSCI.0688-10.2010

17. Qiandong W, Li L, Qiang Z, Fang F, Xiaobing Z, Li Y. Eye avoidance in young children with autism spectrum disorder is modulated by emotional facial expressions. J Abnorm Psychol. 2018;127(7):722-732. doi:10.1037/abn0000372

18. Corden B, Chilvers R, Skuse D. Avoidance of emotionally arousing stimuli predicts social-perceptual impairment in Asperger's syndrome. Neuropsychologia. 2008;46(1):137-147. doi:10.1016/j. neuropsychologia.2007.08.005 
19. Santos A, Silva C, Rosset D, Deruelle C. Just another face in the crowd: evidence for decreased detection of angry faces in children with Williams syndrome. Neuropsychologia. 2010;48(4):1071-1078. doi:10.1016/j.neuropsychologia.2009.12.006

20. Nancy K, Galit Y. The fusiform face area: a cortical region specialized for the perception of faces. Philos Trans $R$ Soc Lond B. 2006;361(1476):2109-2128. doi:10.1098/rstb.2006.1934

21. Dardas LA, Ahmad MM. Coping strategies as mediators and moderators between stress and quality of life among parents of children with autistic disorder. Stress Health. 2015;31(1):5-12. doi:10.1002/ smi. 2513

22. Adolphs R, Spezio ML, Parlier M, Piven J. Distinct face-processing strategies in parents of autistic children. Curr Biol. 2008;18 (14):1090-1093. doi:10.1016/j.cub.2008.06.073

23. Wagner JB, Brandon K, Helen TF, et al. Attentional bias to fearful faces in infants at high risk for autism spectrum disorder. Emotion. 2020;20 (6):980-992. doi:10.1037/emo0000628

24. Nakano T, Tanaka K, Endo Y, et al. Atypical gaze patterns in children and adults with autism spectrum disorders dissociated from developmental changes in gaze behaviour. Proc $R$ Soc B. 2010;277 (1696):2935-2943. doi:10.1098/rspb.2010.0587

25. Vicky T. Eye-tracking study on facial emotion recognition tasks in individuals with high-functioning autism spectrum disorders. Austism. 2018;22(2):161-170. doi:10.1177/1362361316667830

26. Dalton KM, Nacewicz BM, Johnstone T, et al. Gaze fixation and the neural circuitry of face processing in autism. Nat Neurosci. 2005;8 (4):519-526. doi:10.1038/nn1421

27. Santos A, Chaminade T, Fonseca DD, Silva C, Rosset D, Deruelle C. Just another social scene: evidence for decreased attention to negative social scenes in high-functioning autism. J Autism Dev Disord. 2012;42(9):1790-1798. doi:10.1007/s10803-011-1415-6

28. Tang JSY, Chen NTM, Falkmer M, Bölte S, Girdler S, Sonya G. Atypical visual processing but comparable levels of emotion recognition in adults with autism during the processing of social scenes. J Autism Dev Disord. 2019;49(10):4009-4018. doi:10.1007/s10803019-04104-y

29. Shaffer RC, Pedapati EV, Shic F, et al. Brief report: diminished gaze preference for dynamic social interaction scenes in youth with autism spectrum disorders. J Autism Dev Disord. 2017;47(2):506-513. doi:10.1007/s10803-016-2975-2

30. Nayar K, Gordon PC, Martin GE, et al. Links between looking and speaking in autism and first-degree relatives: insights into the expression of genetic liability to autism. BioMed Central. 2018;9(1):51. doi:10.1186/s13229-018-0233-5

31. MJ Wieser, P Pauli, P Weyers, Andreas M. Fear of negative evaluation and the hypervigilance-avoidance hypothesis: an eye-tracking study. $J$ Neural Transm (Vienna). 2009;116(6):717-723. doi:10.1007/s00702-008-0101-0

32. Freeth M, Chapman P, Ropar D, Mitchell P. Do gaze cues in complex scenes capture and direct the attention of high functioning adolescents with ASD? Evidence from eye-tracking. J Autism Dev Disord. 2010;40(5):534-547. doi:10.1007/s10803-009-0893-2

33. Qiandong W, Pan HS, Yuyin W, Man LC, Fang F, Li Y. Gaze response to others' gaze following in children with and without autism. J Abnorm Psychol. 2020;129(3):320-329. doi:10.1037/ abn0000498

34. Zhang L, Sun Y, Chen F, et al. Psychometric properties of the autism-spectrum quotient in both clinical and non-clinical samples: chinese version for mainland China. BMC Psychiatry. 2016;16(1). doi:10.1186/s12888-016-0915-5.

35. Weiss EM, Rominger C, Hofer E, Fink A, Papousek I. Less differentiated facial responses to naturalistic films of another person's emotional expressions in adolescents and adults with highfunctioning autism spectrum disorder. Prog Neuropsychopharmacol Biol Psychiatry. 2019;89:341-346. doi:10.1016/j.pnpbp.2018.10.007
36. Donck SVD, Dzhelyova M, Vettori S, et al. Fast periodic visual stimulation EEG reveals reduced neural sensitivity to fearful faces in children with autism. J Autism Dev Disord. 2019;49(11):46584673. doi:10.1007/s10803-019-04172-0

37. Malaia E, Cockerham D, Rublein K. Visual integration of fear and anger emotional cues by children on the autism spectrum and neurotypical peers: an EEG study. Neuropsychologia. 2019;126:138-146. doi:10.1016/j.neuropsychologia.2017.06.014

38. Ming J, Fs M, Diksha S, Christine C, Qi Z, Suma J. Classifying individuals with ASD through facial emotion recognition and eye-tracking. Annu Int Conf IEEE Eng Med Biol Soc. 2019;2019:6063-6068. doi:10.1109/EMBC.2019.8857005

39. MJ West, DA Copland, WL Arnott, et al. Effects of prosodic and semantic cues on facial emotion recognition in relation to autism-like traits. J Autism Dev Disord. 2018;48(8):2611-2618. doi:10.1007/ s10803-018-3522-0

40. Sucksmith E, Allison C, Baron-Cohen S, Chakrabarti B, Hoekstra RA. Empathy and emotion recognition in people with autism, first-degree relatives, and controls. Neuropsychologia. 2013;51(1):98-105. doi:10.1016/j.neuropsychologia.2012.11.013

41. Tomoko I, Hiroyasu I, Shino O, Nobuo M. Absence of predispositional attentional sensitivity to angry faces in children with autism spectrum disorders. Sci Rep. 2014;4:7525. doi:10.1038/srep07525

42. JS Morris, CD Frith, DI Perrett, et al. A differential neural response in the human amygdala to fearful and happy facial expressions. Nature. 1996;383(6603):812-815. doi:10.1038/383812a0

43. Wm J, Paul P, Ag W, Andreas M. Is eye to eye contact really threatening and avoided in social anxiety?-An eye-tracking and psychophysiology study. $J$ Anxiety Disord. 2009;23(1):93-103. doi:10.1016/j.janxdis.2008.04.004

44. Farabee H, Ramsey C. Social anxiety and speaker gaze in a persuasive atmosphere. Acad Press. 1993;27(4). doi:10.1006/jrpe.1993.1025.

45. Smith ML, Cottrell GW, Gosselin F, Schyns PG. Transmitting and decoding facial expressions. Psychol Sci. 2005;16(3):184-189. doi:10.1111/j.0956-7976.2005.00801.x

46. Gallagher M, Holland PC. The amygdala complex: multiple roles in associative learning and attention.. Proc Natl Acad Sci U S A. 1994;91(25):11771-11776. doi:10.1073/pnas.91.25.11771

47. Adolphs R, Tranel D, Damasio H, Damasio AR. Fear and the human amygdala. $J$ Neurosci. 1995;15(9):5879-5891. doi:10.1523/ JNEUROSCI.15-09-05879.1995

48. LeDoux J. Emotional networks and motor control: a fearful view. Prog Brain Res. 1996;107. doi:10.1016/s0079-6123(08)61880-4

49. Baron-Cohen S, Ring HA, Bullmore ET, Wheelwright S, Ashwin C, Williams SCR. The amygdala theory of autism. Neurosci Biobehav Rev. 2000;24(3):355-364. doi:10.1016/ S0149-7634(00)00011-7

50. Schultz RT. Developmental deficits in social perception in autism: the role of the amygdala and fusiform face area. Int J Develop Neurosci. 2005;23(2-3):125-141. doi:10.1016/j.ijdevneu.2004.12.012

51. Phillips ML, Williams L, Senior C, et al. A differential neural response to threatening and non-threatening negative facial expressions in paranoid and non-paranoid schizophrenics. Psychiatry Res. 1999;92(1):11-31. doi:10.1016/s0925-4927(99)00031-1

52. Whalen PJ, Shin LM, McInerney SC, Fischer H, Wright CI, Rauch SL. A functional MRI study of human amygdala responses to facial expressions of fear versus anger.. Emotion. 2001;1(1):70-83. doi:10.1037/1528-3542.1.1.70

53. Williams LM, Brown KJ, Das $P$, et al. The dynamics of cortico-amygdala and autonomic activity over the experimental time course of fear perception. Brain Res Cogn Brain Res. 2004;21 (1):114-123. doi:10.1016/j.cogbrainres.2004.06.005

54. Zaira C, Susanna S, Carlotta L, et al. Biases in spatial bisection induced by viewing male and female faces. Exp Psychol. 2014;61 (5):368-377. doi:10.1027/1618-3169/a000256 


\section{Publish your work in this journal}

Neuropsychiatric Disease and Treatment is an international, peerreviewed journal of clinical therapeutics and pharmacology focusing on concise rapid reporting of clinical or pre-clinical studies on a range of neuropsychiatric and neurological disorders. This journal is indexed on PubMed Central, the 'PsycINFO' database and CAS, and is the official journal of The International Neuropsychiatric Association (INA). The manuscript management system is completely online and includes a very quick and fair peer-review system, which is all easy to use. Visit http://www.dovepress.com/testimonials.php to read real quotes from published authors.

Submit your manuscript here: https://www.dovepress.com/neuropsychiatric-disease-and-treatment-journal 